\title{
CADERNOS DE PESQUISA INTERDISCIPLINAR EM CIÊNCIAS HUMANAS
}

ISSN 1984-8951

\section{INSTRUÇÕES AOS AUTORES}

\section{A) SUBMISSÃO ELETRÔNICA}

- Manuscritos digitados em WORD, fonte ARIAL, contando de 10 a 35 páginas numeradas e limitando-se a 70.000 (setenta mil) caracteres, incluídos os caracteres em branco. No texto submetido para avaliação NÃO deve constar o nome do autor.

- Margens esquerda e superior de 3,0 cm; direita e inferior de 2,0 cm.

\section{B) DIRETRIZES PARA AUTORES}

\section{Estrutura de Apresentação e Formatação}

\section{PRÉ-TEXTO}

\begin{tabular}{|c|ll|}
\hline & $\bullet$ & Fonte ARIAL \\
& $\bullet$ & Caixa baixa \\
& $\bullet$ & Tamanho 14 \\
Título do & $\bullet$ & Espaçamento simples \\
artigo: & $\bullet$ & Centrado \\
& $\bullet$ & Título em português em negrito \\
& $\bullet$ & Título em inglês em negrito e itálico \\
\hline
\end{tabular}

\begin{tabular}{|l|ll|}
\hline & $\bullet$ & Fonte ARIAL \\
Resumo: & $\bullet$ & Tamanho 12 \\
& $\bullet$ & Espaçamento simples \\
& - & Margem justificada \\
& & Entre 100 e 150 palavras \\
\hline
\end{tabular}




\begin{tabular}{|c|ll|}
\hline & $\bullet$ & Fonte ARIAL \\
Palavras- & $\bullet$ & Tamanho 12 \\
chave: & $\bullet$ & De 3 a 5 palavras \\
& - & Palavras separadas por ponto \\
& - & Primeira letra de cada palavra em caixa alta \\
\hline
\end{tabular}

\begin{tabular}{|l|ll|}
\hline & $\bullet$ & Fonte ARIAL \\
& $\bullet$ & Tamanho 12 \\
Abstract: & $\bullet$ & Espaçamento simples \\
& $\bullet$ & Margem justificada \\
& $\bullet$ & Entre 100 e 150 palavras \\
\hline
\end{tabular}

\begin{tabular}{|l|ll|}
\hline & $\bullet$ & Fonte ARIAL \\
Key Words: & $\begin{array}{ll}\text { - } \\
\bullet\end{array}$ & De 3 a 5 palavras \\
$\bullet$ & Palavras separadas por ponto \\
$\bullet$ & Primeira letra de cada palavra em caixa alta \\
\hline
\end{tabular}

\section{TEXTO}

\begin{tabular}{|l|ll|}
\hline & $\bullet$ & Fonte ARIAL \\
Fonte: & $\bullet$ & Tamanho 12 para o texto \\
& $\bullet$ & Tamanho 10 para citação direta com recuo de $4 \mathrm{~cm}$ \\
\hline
\end{tabular}

\section{Alinhamento: - $\quad$ O texto deve ser justificado}

\begin{tabular}{|c|c|}
\hline Espaçamento: & $\begin{array}{l}\text { - } \quad \text { No texto: } 1,5 \mathrm{~cm} \\
\text { - } \quad \text { Na citação direta com recuo de } 4 \mathrm{~cm} \text { : simples } \\
\text { - } \quad \text { Em notas de rodapé: simples } \\
\text { - } \quad \text { Entre texto e título da seção: } 2 \times 1,5 \mathrm{~cm}\end{array}$ \\
\hline
\end{tabular}

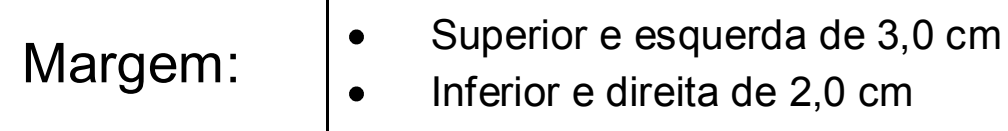




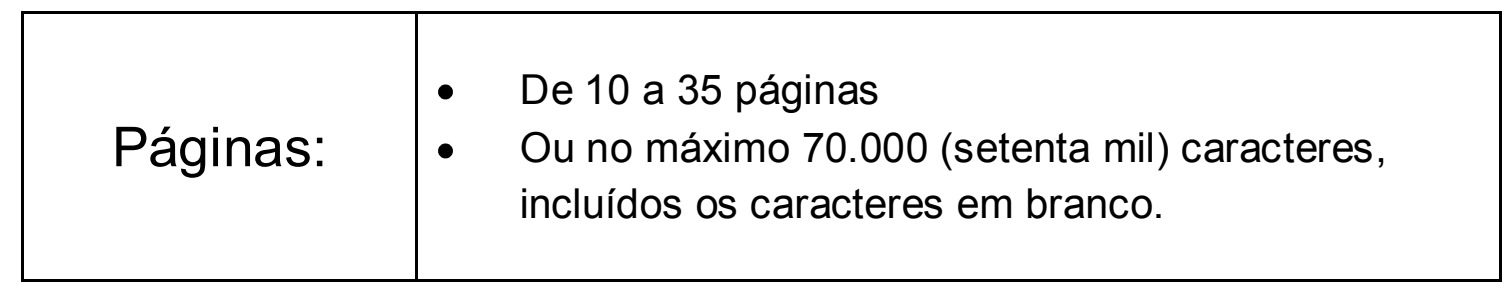

\begin{tabular}{|c|ll|}
\hline & $\bullet$ & Não iniciar uma nova página a cada subtítulo \\
Subtítulos: & $\bullet \begin{array}{l}\text { Os títulos são diferenciados graficamente entre } \\
\text { seções de hierarquia diferentes e iguais quando de } \\
\text { mesma hierarquia }\end{array}$ \\
$\bullet \quad$\begin{tabular}{l} 
Deve seguir uma numeração seqüencial \\
\hline
\end{tabular}
\end{tabular}

\begin{tabular}{|c|ll|}
\hline & $\bullet$ & Fonte ARIAL \\
Notas de & $\bullet$ & Tamanho 10 \\
rodapé: & $\bullet$ & Espaçamento simples \\
& $\bullet$ & Deve ser em número arábico seqüencial \\
\hline
\end{tabular}

\begin{tabular}{|c|c|}
\hline Citação: & $\begin{array}{l}\text { - } \quad \text { Sistema de chamada autor-data. } \\
\text { - } \quad \text { Citações diretas (AUTOR, ano, p.) } \square \text { inclui página } \\
\text { - } \quad \text { duplasos e dentro do texto. } \\
\text { - } \quad \text { Citações diretas com mais de três linhas: sem } \\
\text { aspas, recua a margem esquerda } 4 \mathrm{~cm} \text {, } \\
\text { espaçamento simples, fonte tamanho } 10 . \\
\text { - Citações parafraseadas (AUTOR, ano) } \square \text { não inclui } \\
\text { página }\end{array}$ \\
\hline
\end{tabular}

\begin{tabular}{|c|c|}
\hline \multicolumn{2}{|r|}{ Exemplos de citações } \\
\hline Com um autor & $\begin{array}{l}\text { - Segundo Bauman (1999, p.10), “a ambivalência é } \\
\text { [...]". } \\
\text { • "A ambivalência é [...]" (BAUMAN, 1999, p.10). }\end{array}$ \\
\hline $\begin{array}{c}\text { Com dois ou três } \\
\text { autores }\end{array}$ & $\begin{array}{l}\text { - Segundo Giddens, Beck e Lash (1997, p.38), } \\
\text { "[...]". } \\
\text { • “A modernização é [...]" (GIDDENS; BECK; } \\
\text { LASH, 1997, p.38). }\end{array}$ \\
\hline
\end{tabular}




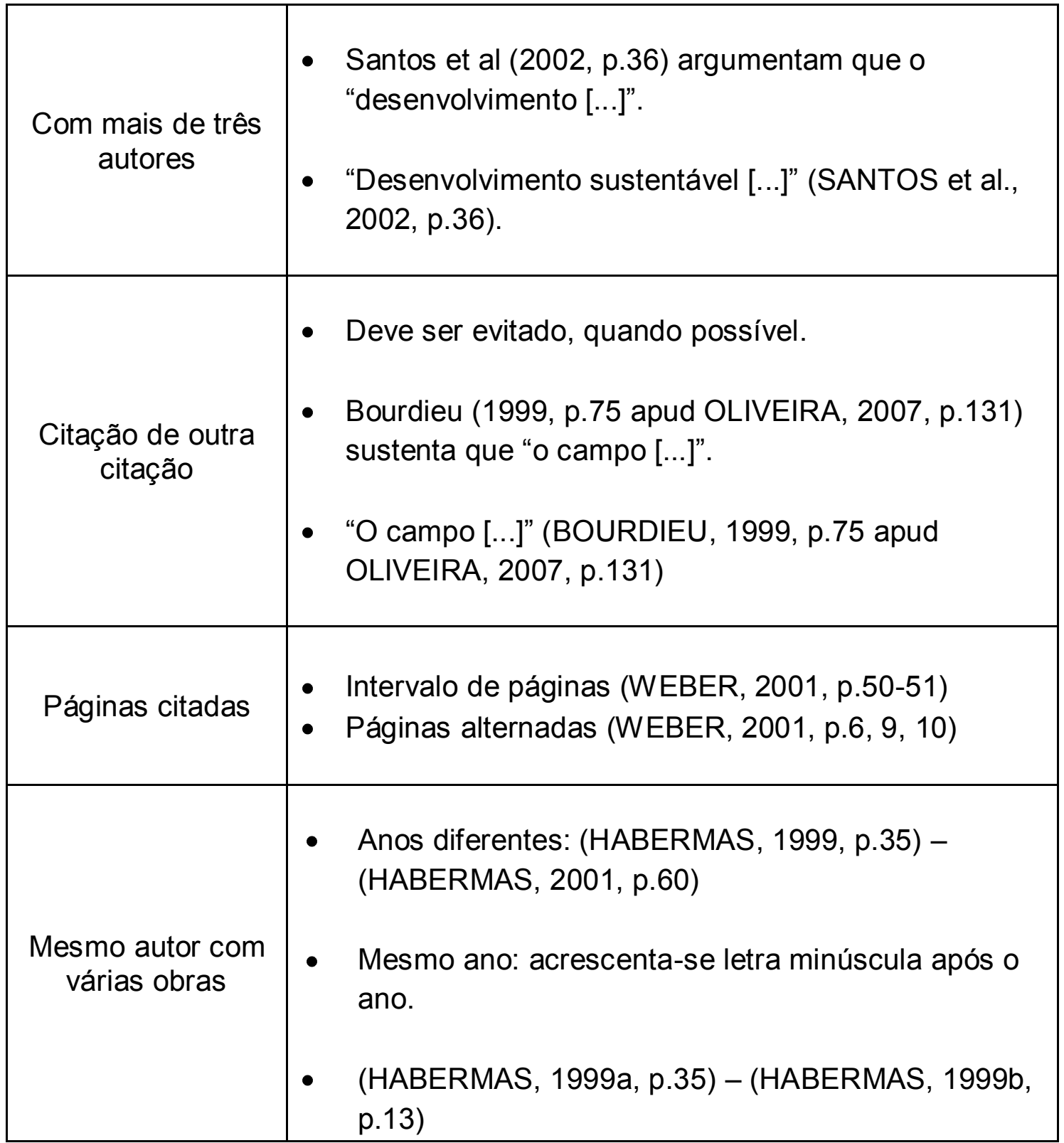

\section{PÓS-TEXTO}

\begin{tabular}{|c|ll|}
\hline & $\bullet$ & Iniciar nova página \\
& $\bullet$ & Fonte ARIAL \\
Título da & $\bullet$ & Tamanho 12 \\
Referência: & $\bullet$ & Deve constar apenas REFERÊNCIAS \\
& $\bullet$ & Centrado \\
& $\bullet$ & Negrito \\
\hline
\end{tabular}




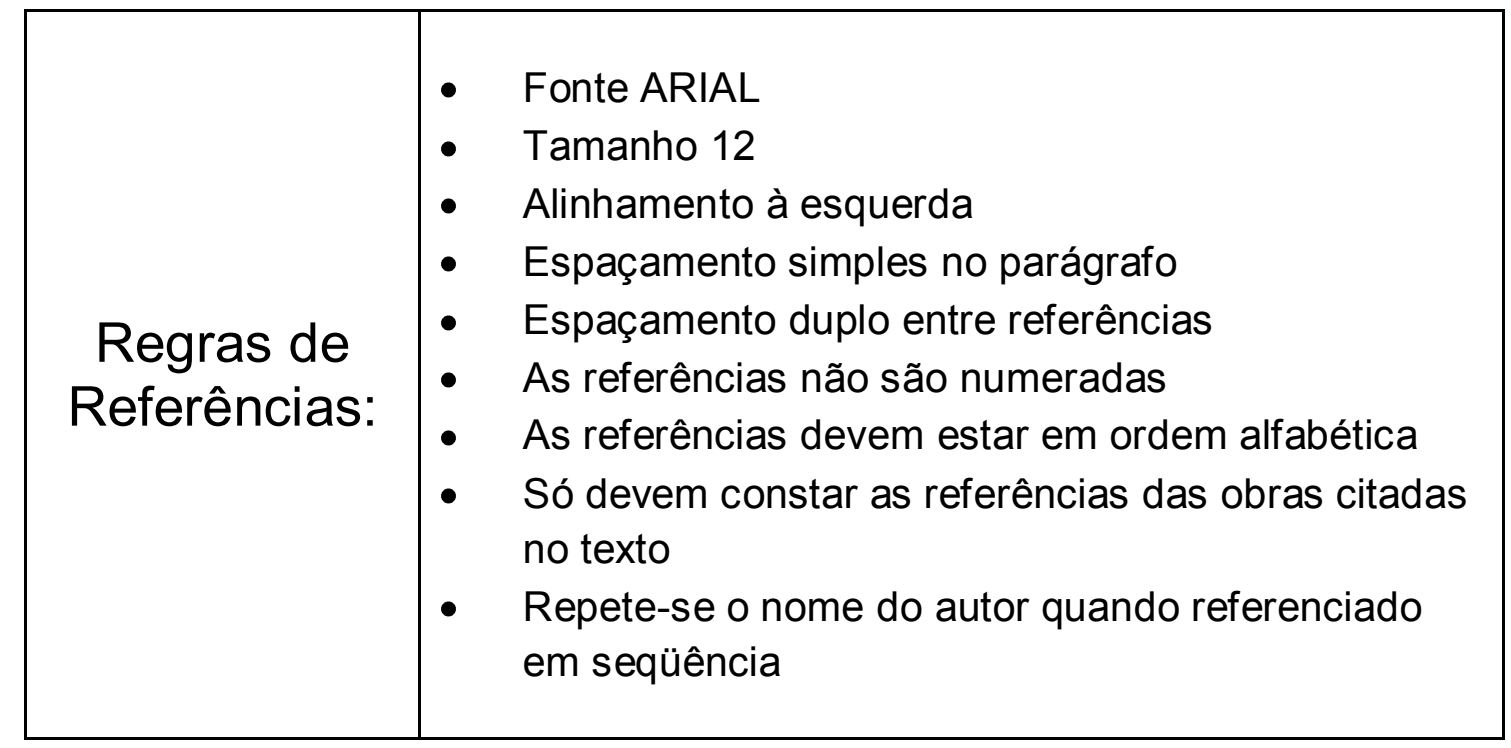

\section{Exemplos de Referências}

a) Livro

Quando há apenas um autor:

FERNANDES, F. Fundamentos empíricos da explicação sociológica. 2. ed. São Paulo: Nacional, 1967.

Quando houver dois ou três autores:

SILVA, F.; FERREIRA, L. P. Globalização no século XXI. São Paulo: Macuco, 2000.

CASTILLO, G.; KOSTOF, S.; TOBIAS, R. A history of architecture: settings and rituals. Oxford: Oxford University Press, 1995.

Quando houver mais de três autores:

MAGAlhãeS, A. D. F. et al. Perícia contábil. 3. ed. São Paulo: Atlas, 2001.

Quando houver organizador (Org.), coordenador (Coord.) ou editor (Ed.):

BOSI, A. (Org.). O conto brasileiro contemporâneo. 6. ed. São Paulo: Cultrix, 1989. 


\section{Quando não há o nome da editora:}

VALÊNCIA, I. Das mulheres e das flores. Belo Horizonte: [s. n.], 1974.

Quando não há data da edição:

SHAKESPEARE, W. Hamleto: Príncipe da Dinamarca. Tradução Carlos Alberto Nunes. São Paulo: Melhoramentos, [s.d.].

Quando houver tradutor, prefácio ou notas:

ALIGHIERI, D. A divina comédia. Tradução Hernani Donato. São Paulo: Círculo do Livro, 1983.

GROTOWSKI, J. Em busca de um teatro pobre. Tradução Aldomar Conrado. Prefácio Peter Brook. Rio de Janeiro: Civilização Brasileira, 1992.

Quando o autor for uma entidade:

ASSOCIAÇÃO BRASILEIRA DE NORMAS TÉCNICAS. NBR 6023:

Informação e documentação. Rio de Janeiro: ABNT, 2000. 3 p.

Quando a obra tiver título e subtítulo:

CERTEAU, M. de. Histoire et psychanalyse: entre science et fiction. Paris: Gallimard, 1987.

\section{b) Capítulo de Livro}

Partes de livro sem autoria especial:

SANTOS, J. R. dos. Avaliação econômica de empresas. In:

Técnicas de análise financeira. 6. ed. São Paulo: Macuco, 2001. p.78-90.

Partes de livro com autoria especial:

ROSA, C. Solução para a desigualdade. In: SILVA, F. (Org.). Como estabelecer os parâmetros da globalização. 2. ed. São Paulo: Macuco, 1999. p.35-48.

CHAUÍ, M. Notas sobre cultura popular. In: OLIVEIRA, P. S. (Org.).

Metodologia das ciências humanas. São Paulo: Hucitec; UNESP, 1998. p.165-182. 


\section{c) Artigo em Periódico}

ALETTI, M. A figura da ilusão na literatura psicanalítica da religião.

Psicologia USP, v.15, n.3, p.163-190, jan./jun. 2004.

OLIVEIRA, A. da C. Considerações constitucionais sobre a pesquisa e aplicação terapêutica das células-tronco. Revista de Direito Privado, São Paulo, ano 8, v.30, p.49-74, abr./jun. 2007.

ESPOSITO, I. et al. Repercussões da fadiga psíquica no trabalho e na empresa. Revista Brasileira de Saúde Ocupacional, São Paulo, v.8, n.32, p.37-45, out./dez. 1979.

RAUD, C. Análise crítica da sociologia econômica de Mark Granovetter: os limites de uma leitura do mercado em termos de redes e imbricação. Política \& Sociedade, Florianópolis, n.6, p.59-82, abr. 2005.

\section{d) Monografia, Dissertação e Tese}

\section{Monografia}

MEDEIROS, J. B. Alucinação e magia na arte. 1993. 86 f. Monografia (apresentada ao final do curso de pós-graduação stricto sensu em Letras) Faculdade de Filosofia, Letras e Ciências Humanas, Universidade de São Paulo, São Paulo.

\section{Dissertação de Mestrado}

RODRIGUES, M. V. Qualidade de vida no trabalho. 1989. $180 \mathrm{f}$. Dissertação (Mestrado em Administração) - Faculdade de Ciências Econômicas, Universidade Federal de Minas Gerais, Belo Horizonte, 1989.

Tese

SOUZA, Zenira Pires de. A responsabilidade social empresarial sob uma perspectiva sistêmica. 2004. 250 f. Tese (Doutorado) - Universidade Federal de Santa Catarina, Centro Tecnológico. Programa de PósGraduação em Engenharia de Produção, Florianópolis, 2004. 


\begin{tabular}{|l|}
\hline e) Eventos \\
\hline Encontro Anual \\
SOARES, T. Empresas estatais privatizadas. In: ENCONTRO ANUAL DA \\
ASSOCIAÇÃO NACIONAL DE PÓS-GRADUAÇÃO EM ADMINISTRAÇÃO, \\
20, 1996, Rio de Janeiro. Anais... Rio de Janeiro: ANPAD, 1996. \\
\hline
\end{tabular}

\begin{tabular}{l} 
f) Internet \\
\hline Artigo de Internet com autor: \\
MALOFF, J. A internet e o seu valor. Ciência da Informação, Brasília, v.26, \\
n.3, 1997. Disponível em: <http://www.ibict.br/cionline/>. Acesso em: 18 out. \\
1998. \\
Artigo de Internet sem autor especial: \\
CIÊNCIA DA INFORMAÇÃO, Brasília, v.26. n.3, 1997. Disponível em : \\
<http://www.ibict.br/cionline/>. Acesso em: 19 maio 1998. \\
Livro em meio eletrônico: \\
ALVES, C. Navio negreiro. [S.I.]: Virtual Books, 2000. Disponível em: \\
<http://www.terra.com.br/virtualbooks/port//port/navionegreiro.htm>. Acesso \\
em: 05 mar. 2004 \\
Simpósios e Congressos em meio eletrônico: \\
ENCONTRO ANUAL DA ASSOCIAÇÃO NACIONAL DE PÓS-GRADUAÇÃO \\
EM ADMINISTRAÇÃO, 20, 1996, Rio de Janeiro. Anais eletrônicos... Rio de \\
Janeiro: ANPAD, 1996. Disponível em: \\
<http://www.anpad.com.br/xxcongresso.anais.htm>. Acesso em: 5 mar. 1997.
\end{tabular}




\begin{tabular}{|c|l|}
\hline \multicolumn{2}{|c|}{ ILUSTRAÇÕES } \\
\hline $\begin{array}{c}\text { Quadros, } \\
\text { desenhos, }\end{array}$ & $\bullet \begin{array}{l}\text { Funcionam como explicações visuais. } \\
\text { figuras, }\end{array}$ \\
$\begin{array}{l}\text { Fotografias devem ser apresentadas } \\
\text { preferencialmente com extensão TIFF. } \\
\text { fotografias, } \\
\text { gráficos, tabelas, } \\
\text { etc. }\end{array}$ & $\bullet \begin{array}{l}\text { Devem ser numeradas em seqüência, com os } \\
\text { títulos e menções de fontes preferencialmente } \\
\text { na parte inferior da ilustração. } \\
\text { Observar os exemplos a seguir: }\end{array}$ \\
\hline
\end{tabular}

\begin{tabular}{ccccc}
\hline Sexo & $\mathbf{2 0 0 4}$ & $\mathbf{2 0 0 5}$ & $\mathbf{2 0 0 6}$ & $\mathbf{2 0 0 7}$ \\
\hline Masculino & $88,8 \%$ & $88,9 \%$ & $92,5 \%$ & $90,7 \%$ \\
Feminino & $11,2 \%$ & $11,1 \%$ & $7,5 \%$ & $9,3 \%$ \\
\hline
\end{tabular}

Tabela 1: Distribuição percentual da população ocupada na indústria por gênero (2004- 2007)

Fonte: IBGE/PNAD, 2008.

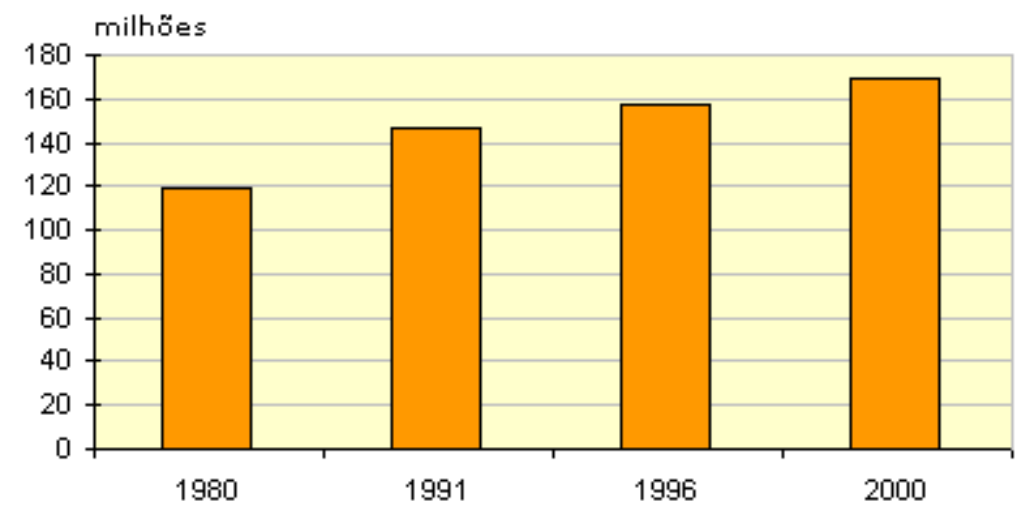

Gráfico 1: População total no Brasil (1980-2000).

Fonte: IBGE, 2007. 